\title{
Was sich bei der Inszenierung von Fremdsprache im Gehirn abspielt
}

\author{
Sambanis, Michaela (2013): Fremdsprachenunterricht und \\ Neurowissenschaften. Tübingen: Narr. ISBN: \\ 978-3-8233-6800-7
}

\section{Manfred Schewe Micha Fleiner}

Es ist eine ebenso facetten- wie beziehungsreiche Trias aus Sprache, Lernen und Gehirn, die den inhaltlichen Rahmen der vorliegenden Monografie Fremdsprachenunterricht und Neurowissenschaften bildet: Mit Bezug auf eine lehrorientierte Zielleserschaft stellt sich Michaela Sambanis der Herausforderung, Prozesse und Kontexte des fremdsprachlichen Lernens aus einer neurowissenschaftlich motivierten Perspektive aufzuzeigen. Hierbei zieht die Verfasserin eine übergreifende Akzentlinie um die Themenfelder Wie Lernen das Gehirn verändert (Kapitel 1), Emotionen und Lernen im Fremdsprachenunterricht (Kapitel 2), Teenager als besondere Fremdsprachenlerner (Kapitel 3) und - für SCENARIO-Leserinnen und Leser gewiss von besonderem Interesse Bewegung und Lernen (Kapitel 4) sowie Dramapädagogik (Kapitel 5).

Im Fokus des Eröffnungskapitels steht die Frage nach Zusammenhängen zwischen Gehirnaktivitäten und menschlichen Lernvorgängen: Hier erhalten die Lesenden theoretisch vertiefte - stets auf dem aktuellen Kenntnisstand der Hirnforschung fußende - Einblicke in zentrale Strukturen und Funktionsweisen des Gehirns. Angesichts des hohen theoriegestützten Informationsgehaltes wäre es in diesem Abschnitt von Vorteil gewesen, der anvisierten Lesergruppe - Studierende, Lehrende, Auszubildende - ergänzende Visualisierungen an die Hand zu geben, um den (Erst)zugang zu neurowissenschaftlichen Sachverhalten zu erleichtern. Wertvolle Dienste wiederum leisten die am Ende eines jeden Kapitels platzierten Praxisfenster, in denen Kerninhalte aufgegriffen und - in Gestalt eines fiktiven Diskussionsforums - auf unterschiedliche Formate der Schulpraxis übertragen werden.

Der bewusste Umgang mit Lernenden im Teenageralter (Kapitel 3) sowie das dynamische Ineinandergreifen von Ratio und Emotio in zielsprachlichen LehrLern-Prozessen (Kapitel 2) bilden die Schwerpunkte der beiden Folgekapitel. Die konsequent im Themenfeld des Fremdsprachenunterrichts verankerten Ausführungen überzeugen nicht allein durch aufschlussreichen Gehalt, sondern auch durch einen pointierten, erfrischend kurzweiligen Schreibstil. Als erfahrene Didaktikerin achtet Sambanis hierbei sorgfältig darauf, den Lesenden komplexe Sachverhalte gut verständlich und in ansprechender Form zu vermitteln. 
Kapitel 4 ist dem Thema Bewegung und Lernen gewidmet. Die Autorin erinnert anfangs daran, dass man sich bereits in der Antike einer lernförderlichen Wirkung von Bewegung bewusst war, um danach verschiedene Gründe für bewegtes Lernen aufzulisten und jeweils kurz zu kommentieren (S. 89):

- Anthropologische Gründe (Bewegung = Grundbedürfnis)

- Gesundheitserzieherische Gründe (Bewegung = Verbesserung des physischen und psychischen Wohlergehens)

- Motivationale Gründe (Bewegung = Abwechslung, Element des Neuen)

- Lerntheoretische Gründe (Bewegung = Mittel der Informationsverarbeitung)

- Neurophysiologische Gründe (Bewegung = Aktivierung verschiedener Hirnareale)

Mit Blick auf den Fremdsprachenunterricht konzentriert sich die Autorin auf bewegungsbezogene Aktivitäten, die auf eine lernprozessstützende Wirkung zielen. So werden etwa

\begin{abstract}
Bewegung und Inhalt mehrfach im Verbund wiederholt, sodass eine Verknüpfung entsteht. Bewegungen werden hierbei zur Unterstützung des Enkodierungsvorgangs bei der Begegnung mit Neuem, beim Einprägen, Üben und Wiederholen genutzt. Aber auch beim Abrufen scheinen sie stützend zu wirken, d.h. die Erinnerung an die Bewegung kann den Zugriff auf die Information ermöglichen. (S. 93)
\end{abstract}

Sambanis betont, dass Bewegung nicht nur Abwechslung in den LehrLernprozess bringt, sondern Inhalte sowohl besser und länger behalten als auch schneller und zuverlässiger abgerufen werden können. Orientiert man sich am derzeitigen Stand der Forschung, so ergänzt sie, lässt sich zumindest für die Bereiche Wortschatz und Aussprache von einer lernförderlichen Wirkung von Bewegungen auf das Lernen einer Fremdsprache ausgehen. Die Autorin bezieht sich auf konkrete Studien, um zu belegen, dass Lernende, die Bewegung als Hilfsmittel einsetzen, im Vergleich zu „nicht-bewegten Kontrollgruppen“ effektiver lernen. Ihre Exkursionen in die Gehirnforschung sind dabei sehr lehrreich und relativieren etwa die Sicht einer scharfen Trennung zwischen rechter und linker Gehirnhälfte:

Letztlich ist sogar anzunehmen, dass z.B. am Verstehen von gesprochenen Sätzen nicht nur unterschiedliche Regionen der linken Hirnhälfte, sondern beide Hemisphären beteiligt sind, da die Satzmelodie neben Syntax und Semantik wichtige Informationen auf Satzebene transportiert. Melodische und musikalische Merkmale werden aber nicht linkshemisphärisch, sondern bei den meisten Menschen in der rechten Gehirnhälfte verarbeitet. (S. 105) 
Für SCENARIO-Leserinnen und -leser dürfte Kapitel 5 mit dem Titel Dramapädagogik von besonderem Interesse sein. Die Autorin führt hier anfangs in die Grundlagen der Dramapädagogik ein. Sie deckt beispielsweise verschiedene Phasen eines dramapädagogischen Unterrichts ab, bezieht sich auf Aspekte von Lehrerrolle und Feedback und stellt verschiedene Inszenierungsformen/techniken vor. Ihr Versuch einer Unterscheidung zwischen Drama- und Theaterpädagogik gelingt allerdings nicht ganz, denn speziell das Schema auf S. 117 vermittelt den Eindruck, als sei die Dramapädagogik prozess- und die Theaterpädagogik produktorientiert. Das ist eine zu grobe Verallgemeinerung und es bleibt ein Forschungsdesiderat, die Unterschiede zwischen der aus der britischen Tradition stammenden Dramapädagogik und der deutschen Theaterpädagogik bzw. das Gemeinsame dieser Traditionen differenzierter herauszuarbeiten.

In Anknüpfung an die vorherigen Kapitel führt Sambanis allerdings im Schlusskapitel überzeugend zusammen, dass aus neurowissenschaftlicher Sicht viele plausible Gründe für eine dramapädagogische Gestaltung von Fremdsprachenunterricht bestehen. So wird in einem solchen Unterricht etwa eine multimodale Verarbeitung begünstigt und auf Bedeutsamkeit und persönliche Relevanz von Unterrichtshandlungen geachtet. Dramapädagogische Übungen eignen sich dazu, sprachliche Muster und Bewegungen zu koppeln und sogenannte Flow-Erlebnisse zu schaffen, die mit positiven Emotionen verbunden sind und zu einem idealen Fokussierungszustand führen.

Fazit: Neben einer überzeugenden Vernetzung klassischer Themen der Fremdsprachendidaktik mit dem derzeitigen Erkenntnisstand der Neurowissenschaften gelingt es Sambanis mit der vorliegenden Publikation, insbesondere im Hinblick auf eine theoretische Fundierung performativer Formen des Lehrens und Lernens einen neuen Meilenstein zu setzen. Sowohl Kolleginnen und Kollegen, die in der Lehreraus- und -fortbildung tätig sind, als auch Lehramtsstudierenden fremdsprachlicher Fächer sei sie daher wärmstens empfohlen. 